\title{
Unconditionally Secure Proactive Secret Sharing Scheme with Combinatorial Structures
}

\author{
Douglas R. Stinson and R. Wei \\ Department of Combinatorics and Optimization \\ University of Waterloo \\ Waterloo, Ontario N2L 3G1, Canada \\ \{dstinson,rwei\}@cacr.math. uwaterloo.ca
}

\begin{abstract}
Verifiable secret sharing schemes (VSS) are secret sharing schemes dealing with possible cheating by the participants. In this paper, we propose a new unconditionally secure VSS. Then we construct a new proactive secret sharing scheme based on that VSS. In a proactive scheme, the shares are periodically renewed so that an adversary cannot get any information about the secret unless he is able to access a specified number of shares in a short time period. Furthermore, we introduce some combinatorial structure into the proactive scheme to make the scheme more efficient. The combinatorial method might also be used to improve some of the previously constructed proactive schemes.
\end{abstract}

\section{Introduction}

One important topic in cryptography is how to securely share a secret among a group of people. In some cases, many people need to share the power to use a cryptosystem. Thus some secret information should be shared by a group so that the cryptosystem can be used only if it is permitted by a specified subset of the group. The study of how to keep a secure backup of a secret key and how to recover it securely has been first studied by Blakley 4 and Shamir 23 independently. Shamir proposed a polynomial threshold scheme. In a $(t, n)$ threshold scheme, a secret value is shared by $n$ participants such that any $t$ of the participants can reconstruct the secret value by putting their shares together, but any $t-1$ participants cannot get any information about the secret value. In such a scheme, an adversary needs to compromise at least $t$ locations in order to learn the secret, and corrupt at least $n-t-1$ locations to destroy the secret.

In many situations, such as cryptographic master keys, data files, legal documents, etc., a secret value needs to be stored for a long time. In these situations, an adversary may attack the locations one by one and eventually get the secret or destroy it. To prevent such an attack, proactive secret sharing schemes are proposed. Proactive security for secret sharing was first suggested by Ostrovsky and Yung in 18 . In 18 they presented, among other things, a proactive polynomial secret sharing scheme. Proactive security refers to security and availability in the the presence of a mobile adversary. Herzberg et al. I.5 specialized this notion to robust secret sharing schemes and gave a detailed efficient proactive 
secret sharing scheme. In their scheme, a secret value is shared by $n$ servers. The mobile adversary is able to attack all the servers during a long period of time. However, since the corrupted servers can be rebooted, in any time period there are only a subset of servers that are corrupted. "Robust" means that in any time period, the servers can reconstruct the secret value correctly.

The scheme in 15 is based on Shamir's polynomial threshold scheme, thus most aspects of the scheme are unconditionally secure. However, their scheme depends on verifiable secret sharing schemes based on 9 I9 which depend on some cryptographic assumptions. The security of the scheme in $\mathrm{g}$ is based on the hardness of solving discrete logarithm. In the scheme of [I], the privacy of the secret is unconditionally secure, but the correctness of the shares depends on a computational assumption. In a sense, these two schemes complement each other.

The purpose of this paper is to provide a new proactive secret sharing scheme which is unconditionally secure, i.e., the security of any part of the scheme is not based on any cryptographic assumption. Let $S$ be the set of possible secret values, where $|S|=q$. Then unconditional security of the scheme means that at any time the adversary cannot guess the shared secret $s \in S$ with probability better than $\frac{1}{q}$.

We first propose an unconditionally secure verifiable secret sharing scheme. This scheme has some similar features to the absolute VSS in 3. Then we propose several protocols to make it proactive. Following from the method of I.5, the lifetime of the secret is divided into periods of time in the proactive scheme. In each time period, the $n$ shares will be renewed while the secret remains the same. In this way, a mobile adversary who is able to attack (learn or corrupt) at most $b$ shares in a time period cannot learn any information about the secret in the long lifetime. This scheme is also robust, i.e., the secret can be reconstructed at any time.

Furthermore, we introduce some combinatorial structures in the scheme so that the scheme will be more efficient. With the combinatorial structure, most of the computation of the system will depend on the parameter $b$. Thus there is a "trade-off" between the computation and the value of $b$ : when $b$ is smaller (the ability of the adversary is more limited), the computation takes less time. Thus our scheme is more efficient in the situation when the number of the possible corrupted servers are much smaller as compared to the total number of the servers in the system. On the other hand, our combinatorial method might be easily adapted to the scheme of 15 to make the scheme more efficient.

The rest of this paper is arranged as follows. In Section 2 we give some preliminaries and the main settings of the system. Section 3 describes our new verifiable secret sharing scheme. We also propose an anonymous VSS in a subsection. Section 4 describes the proactive scheme without combinatorial structure. Section 5 introduces the combinatorial structure and describes how to apply it to the proactive scheme. 


\section{Preliminaries}

\subsection{Previous Work}

Proactive refers to the security of the scheme in the presence of a mobile adversary who may corrupt all participants of the scheme throughout the lifetime of the system but cannot corrupt too many participants during any short period of time. Such a mobile adversary was first considered by Ostrovsky and Yung in 18.

The motivation of 18 is to combat mobile viruses. The scheme requires the participants to constantly exchange messages and to be able to erase parts of its memory. A polynomial secret sharing proactive scheme is proposed which uses the verifiable secret sharing scheme of 22 .

Herzberg et al. 15 further discussed proactive secret sharing schemes and gave a detailed practical scheme. In their scheme the lifetime is divided into periods of time. At the beginning of each time period, the share holders engage in an interactive update protocol which includes a share recovery protocol and a share renewal protocol. At the end of the period, each shareholder holds completely new shares of the same secret. The secret will not be computed during the update phase while it can be reconstructed at any time. They used the polynomial-based method from 18 for the renewal protocol. They also proposed a polynomialbased method for share recovery protocol. The verifiable secret sharing schemes they used are from 919 .

There are also many papers that discuss proactive security, see e.g., 6141121 and their references. Our discussion will mainly follow the papers 1518 .

\subsection{The Setting}

We will follow the setting of the scheme in 1518 . We assume that there is a system of $n$ servers $P_{1}, P_{2}, \cdots, P_{n}$, which are connected to a common broadcast channel such that messages sent through this channel instantly reach every server. We also assume that the system is synchronized, i.e., the servers can access a common global clock, and that each server has a local source of randomness. To make things simpler, we assume that there are private channels between each pair of servers and that messages sent by broadcast are safely authenticated. With these assumptions, we are able to focus on the proactive scheme itself.

There is an adversary which can corrupt $b$ servers during any time period. Corrupting a server means learning the secret information in the server, modifying its data, sending out wrong message, changing the intended behavior of the server, disconnecting it, and so on. Since the server can be rebooted, the adversary is a mobile one.

A secret value $s \in G F(q)$ will be shared by the servers through the scheme. The value of $s$ needs to be maintained for a long period of time. The life time is divided into time periods which are determined by the global clock. At the 
beginning of each time period the servers engage in an interactive update protocol. The update protocol will not reveal the value of $s$. At the end of the period the servers hold new shares of $s$. The mobile adversary who corrupts $b$ servers in a time period cannot get any information about the secret value $s$. The system can reproduce $s$ in the presence of the mobile adversary at any time.

We consider unconditional security in this paper, which means that the adversary cannot guess the secret with probability better than $\frac{1}{q}$ if the secret $s \in G F(q)$.

\section{Verifiable Secret Sharing}

Since secret sharing schemes were proposed initially by Shamir 23 and Blakley 4, research on this topic has been extensive. In the "classic" secret sharing schemes, there are assumed to be no faults in the system. Tompa and Woll 26, and McEliece and Sarwate 17 first considered schemes with faulty participants and gave partial solutions for that problem. In their schemes, the dealer is always assumed honest. Chor et al. $\varangle$ first defined the complete notion of Verifiable Secret Sharing (VSS), and gave a solution which is based on some cryptographic assumption. In a VSS, each holder of a share can verify that the share is consistent with the other shares. Thus both the dealer and other participants can be verified in such a scheme. There are two aspects of the security in a VSS. One is the security of the secret and the other is the security of the verification.

There are many papers which have discussed VSS recently. Most schemes use

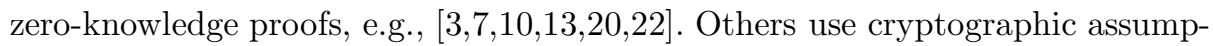
tions such as the hardness of discrete logarithm, see 917.12 proposed a simple and efficient VSS, but it based on some "collision resistance" assumption. On the other hand, many known VSS are not easy to adapt for proactive property.

The VSS in 22 199 are used in proactive schemes in 18 15. 18 used the VSS from 22 which used some zero-knowledge proofs. 15 used the VSS of Feldman 9 and Pedersen 19. The security of the scheme in 9 is based on the hardness of solving discrete logarithm. In the scheme of 19 , the privacy of the secret is unconditionally secure, but the verification depends on a computational assumption.

In 3 it was shown that in any unconditionally secure VSS, $b<\frac{n}{3}$. Thus the VSS with $b \geq \frac{n}{3}$ will either depend on some cryptographic assumption or have small probability of errors. In this section, we will propose an unconditionally secure VSS with $b \leq \frac{n}{4}-1$, which is simpler and more efficient than the scheme in 3. Moreover, our scheme has the threshold property that any coalition of $t-1$ participants cannot get any information about the secret value (regardless of whether the coalition consists of good or bad participants), a property which the scheme of 3 does not have, since secret information may be revealed during the "share" protocol. Another feature of our scheme is that it requires less secret information to be communicated by the dealer, and the dealer is not required to take part in the protocol after the initial distribution of secret information. 


\subsection{Definition}

Now we give a formal definition of VSS, as follows.

Suppose there are a dealer $D$ and $n$ other participants $P_{1}, P_{2}, \cdots, P_{n}$ all connected by private communication channels. They also have access to a broadcast channel. There is a static adversary $A$ that can corrupt up to $b$ of the participants including the $D$. Here static means that the $b$ participants controlled by the adversary are fixed.

Let $\pi$ be a protocol consisting of two phases Share and Reconstruct. Let $S$ be the set of possible secret values. At the beginning of Share, the dealer inputs a secret $s \in S$. At the end of Share each participant $P_{i}$ is instructed to output a Boolean value $v_{e r}$. At the end of Reconstruct each participant is instructed to output a value in $S$.

The protocol $\pi$ is an unconditionally secure Verifiable Secret Sharing protocol if the following properties are hold:

1. If a good player $P_{i}$ outputs ver $_{i}=0$ at the end of Share then every good player outputs ver $_{i}=0$;

2. If the dealer is good, then $v e r_{i}=1$ for every good $P_{i}$.

3. If at least $n-b$ players $P_{i}$ output $v e r_{i}=1$ at the end of Share, then there exists an $s^{\prime} \in S$ such that the event that all good $P_{i}$ output $s^{\prime}$ at the end of Reconstruct is fixed at the end of Share and $s^{\prime}=s$ if the dealer is good;

4. If $|S|=q$ and $s$ is chosen randomly from $S$, and the dealer is good, then any coalition of at most $t-1$ participants cannot guess at the end of Share the value $s$ with probability better than $\frac{1}{q}$.

\subsection{The New VSS}

In this subsection we provide a new unconditionally secure VSS which will be used in our proactive scheme later.

Suppose there is a dealer $\mathrm{D}$ and $n$ participants $P_{i}, 1 \leq i \leq n$, where $n \geq t+3 b$ and $t>b$. Let $S=G F(q)$ be a finite field and let $\omega$ be a primitive element in $G F(q)$. In the following protocol, all the computations are in the field $G F(q)$. We first state the share phase as follows.

\section{Share}

1. When D wants to share a secret value $s \in S$, he chooses a random symmetric polynomial

$$
f(x, y)=\sum_{i=0}^{t-1} \sum_{j=0}^{t-1} a_{i j} x^{i} y^{j}
$$

where $a_{00}=s$ and $a_{i j}=a_{j i}$ for all $i, j$. Then, for each $k$, D sends $h_{k}(x)=$ $f\left(x, \omega^{k}\right)$ to $P_{k}$ through a private channel.

2. After receiving $h_{k}(x)$, each $P_{k}$ sends $h_{k}\left(\omega^{l}\right)$ to $P_{l}$ for $1 \leq l \leq n,(l \neq k)$ through a private channel. 
3. Each $P_{l}$ checks whether $h_{k}\left(\omega^{l}\right)=h_{l}\left(\omega^{k}\right)$ for $1 \leq k \leq n,(l \neq k)$. If $P_{l}$ finds that $h_{k}\left(\omega^{l}\right) \neq h_{l}\left(\omega^{k}\right)$, then $P_{l}$ broadcasts $(l, k)$.

4. Each $P_{i}$ computes the maximum subset $G \subseteq\{1, \cdots, n\}$ such that any ordered pair $(l, k) \in G \times G$ is not broadcasted. If $|G| \geq n-b$, then $P_{i}$ outputs eer $_{i}=1$. Otherwise, $P_{i}$ outputs ver $_{i}=0$.

It is obvious that every good participant computes the same subset $G$ in the end of Share. Next we consider the reconstruct phase. Note that although the adversary is static, he could provide correct information in Share phase but wrong information in Reconstruct phase.

\section{Reconstruct}

1. Each $P_{i}$ sends $h_{i}(0)$ to $P_{k}$, where $i \in G$.

2. After receiving $h_{i}(0), P_{k}$ computes a polynomial $f_{k}(0, y)$ such that $f_{k}\left(0, \omega^{i}\right)=$ $h_{i}(0)$ for at least $n-2 b$ of the data he received. This can be done efficiently using methods of 24 .

3. $P_{k}$ computes and output $s^{\prime}=f_{k}(0,0)$.

In order to prove that the protocol is an unconditionally secure VSS, we need the following lemma.

Lemma 1 Suppose there are $T$ polynomials $h_{1}(x), h_{2}(x), \cdots, h_{T}(x)$ with degree at most $t-1$, where $T \geq t$, such that $h_{i}\left(\omega^{j}\right)=h_{j}\left(\omega^{i}\right)$ for all $i, j$. Then there exists a polynomial $h(x)$ of degree at most $t-1$ such that $h\left(\omega^{i}\right)=h_{i}(0)$ for all $i, 1 \leq i \leq T$. Equivalently, any $t$ of the shares $h_{i}(0), 1 \leq i \leq T$, determine the same secret $K=h(0)$.

Proof First we note that for any $t$-subset $I=\left\{i_{1}, i_{2}, \cdots, i_{t}\right\} \subseteq\{1,2, \cdots, T\}$ and any $h_{j}(x)$, where $1 \leq j \leq T$, we can use the Lagrange interpolation formula (see 25 ) to compute

$$
h_{j}(0)=\sum_{i \in I} h_{j}\left(\omega^{i}\right) b_{i}=\sum_{i \in I} h_{i}\left(\omega^{j}\right) b_{i},
$$

where

$$
b_{i}=\prod_{k \in I, k \neq j} \frac{\omega^{k}}{\omega^{k}-\omega^{j}}
$$

$1 \leq i \leq t$. This comes from the condition $h_{i}\left(\omega^{j}\right)=h_{j}\left(\omega^{i}\right)$ for any $i, j \in$ $\{1,2, \cdots, T\}$.

Now suppose that $I$ and $J$ are two different $t$-subsets of $\{1,2, \cdots, T\}$. Then we can compute a polynomial $h_{I}(x)$ such that $h_{I}\left(\omega^{i}\right)=h_{i}(0)$ for all $i \in I$, and then $h_{I}(0)$ can be obtained by the Lagrange interpolation:

$$
h_{I}(0)=\sum_{i \in I} h_{i}(0) b_{i} .
$$


By the above discussion we have

$$
\begin{aligned}
h_{I}(0) & =\sum_{i \in I} b_{i} h_{i}(0) \\
& =\sum_{i \in I} b_{i} \sum_{j \in J} h_{i}\left(\omega^{j}\right) b_{j} \\
& =\sum_{j \in J} b_{j} \sum_{i \in I} h_{i}\left(\omega^{j}\right) b_{i} \\
& =\sum_{j \in J} b_{j} h_{j}(0) \\
& =h_{J}(0) .
\end{aligned}
$$

We are now in a position to prove the following theorem.

Theorem 2 The scheme of this section is an unconditionally secure verifiable secret sharing scheme.

Proof We prove that the above scheme satisfies the conditions of the VSS as follows.

1. If a good player $P_{i}$ outputs $v^{2} r_{i}=0$, then the size of the maximum subset $G$ is at most $n-b-1$. Thus every good player will output " 0 ".

2. If the dealer is good, then the good player receives $f\left(x, \omega^{i}\right)$. Since $f(x, y)$ is symmetric, $f\left(\omega^{l}, \omega^{i}\right)=f\left(\omega^{i}, \omega^{l}\right)$ for all good players $P_{l}$. Thus all good players are in the subset $G$. Therefore $v^{2} r_{i}=1$ for each good player $P_{i}$.

3. Suppose at least $n-b$ players output " 1 " at the end of the Share. Then there is a subset $G$ of size $n-b$ such that no one in the subset complained the others. Since we assume that there are at most $b$ bad players, there are at least $n-2 b$ good players in $G$, who all have consistent shares. By Lemma II any $t$-subset of the good players can compute the same value $K$. It is easy to check that if the dealer is good, then we have $K=s$, the secret value. Further at most $b$ out of the $n-b$ shares in $G$ are not consistent with the secret $K$. Since $n-b \geq t+2 b$, the algorithms in 24 can be used to find the maximum consistent set of shares and thus determine $K$.

4. Without loss of generality, we assume that the coalition knows the values of $h_{1}(x), h_{2}(x), \cdots, h_{t-1}(x)$. It is easy to show (see, e.g., 5) that for any value $s^{\prime} \in G F(q)$, we can find $b_{i j} \in G F(q)$, where $b_{00}=s^{\prime}, b_{i j}=b_{j i}, 0 \leq i, j \leq t-1$ such that if

$$
f^{\prime}(x, y)=\sum_{i=0}^{t-1} \sum_{j=0}^{t-1} b_{i j} x^{i} y^{j},
$$

then $f^{\prime}\left(x, \omega^{k}\right)=h_{k}(x)$ for $k=1,2, \cdots, b$.

Remark. This scheme is modified from Blom's key predistribution scheme (see 5 for the details). For simplicity, our description used a Reed-Solomon code 
instead of general MDS codes. It is straightforward to generalize our scheme by using MDS codes.

\subsection{An Example}

We display a toy example in this subsection. Let $q=13, \omega=2, n=9, t=3$ and $b=2$. First suppose the dealer $D$ is good. First $D$ selects a polynomial as follows:

$$
f(x, y)=3+9 x+2 x^{2}+9 y+2 y^{2}+8 x y+11 x y^{2}+11 x^{2} y+4 x^{2} y^{2} .
$$

Then $D$ sends the vector $\left(v_{1}, v_{2}, v_{3}\right)$ to the players as follows, each of which determines a polynomial $v_{1}+v_{2} x+v_{3} x^{2}$ :

$$
\begin{aligned}
& h_{1} \longleftarrow(3,4,1) \\
& h_{2} \longleftarrow(6,9,6) \\
& h_{3} \longleftarrow(8,10,8) \\
& h_{4} \longleftarrow(9,2,6) \\
& h_{5} \longleftarrow(12,11,4) \\
& h_{6} \longleftarrow(9,12,8) \\
& h_{7} \longleftarrow(6,11,9) \\
& h_{8} \longleftarrow(12,10,10) \\
& h_{9} \longleftarrow(7,12,1)
\end{aligned}
$$

Suppose that only $P_{1}$ and $P_{2}$ are bad and send wrong data to the other players. Then the pairs broadcasted are of the form $(1, i),(2, i),(i, 1)$ or $(i, 2)$. So the good players will find $G=\{3,4,5,6,7,8,9\}$ and output "1". Since we assume that there are at most 2 bad players, all the good players will output " 1 " if the dealer is good. On the other hand, the player $P_{i}$ chooses " 0 " or " 1 " only depending on the broadcasted pairs, so all good players will output the same value of $v^{2} r_{i}$.

Now suppose that there are at least 7 players who output " 1 ". Since there are at most 2 bad players, it is true that the subset $G$ is found. Suppose, for example, $G=\{1,2,3,4,5,6,7\}$. Then all the good players in $G$ possess consistent shares regardless of whether $D$ is good or bad. However up to two of these players may be bad, and send incorrect shares during Reconstruction. Thus in the Reconstruction phase, there are at least 5 consistent shares held by each of the players. Thus each good player will compute the same polynomial $f(x, 0)$ using the methods of 24 .

\subsection{VSS without Dealer}

Secret sharing without dealer means that there is no dealer in the scheme, who knows and distributes the secret. Secret sharing without dealer is first considered in 16 . One such secret sharing scheme is considered in I9.

We can remove the dealer from our scheme as follows. The other properties of the scheme are the same as in the previous subsection.

Share 
1. Each $P_{k}$ chooses an independent random symmetric polynomial

$$
f^{(k)}(x, y)=\sum_{i=0}^{t-1} \sum_{j=0}^{t-1} a_{i j} x^{i} y^{j}
$$

where $a_{00}=s_{k}$ and $a_{i j}=a_{j i}$ for all $i, j$. Then $P_{k}$ sends $h_{l}^{(k)}(x)=f^{(k)}\left(x, \omega^{l}\right)$ to $P_{l}$ through a private channel.

2. After receiving $h_{l}^{(k)}(x)$, each $P_{l}$ sends $h_{l}^{(k)}\left(\omega^{m}\right)$ to $P_{m}$ for $1 \leq m \leq n$ through a private channel.

3. $P_{m}$ checks whether $h_{m}^{(k)}\left(\omega^{l}\right)=h_{l}^{(k)}\left(\omega^{m}\right)$ for $1 \leq l \leq n$. If $P_{m}$ finds that $h_{m}^{(k)}\left(\omega^{l}\right) \neq h_{l}^{(k)}\left(\omega^{m}\right)$, then $P_{m}$ broadcasts $(k ; m, l)$.

4. For every $k \neq m$, each player $P_{m}$ computes the maximum subset $G_{k}$ such that for any pair $(m, l) \in G_{k} \times G_{k},(k ; m, l)$ is not broadcasted. If $\left|G_{k}\right| \geq n-b$, then $P_{m}$ puts the value $k$ in a list $\mathcal{L}$.

5. If $|\mathcal{L}| \geq n-b$, then $P_{m}$ outputs ver $_{m}=1$ and computes his share as

$$
h_{m}=\sum_{l \in \mathcal{L}} h_{m}^{(l)}(x) .
$$

Otherwise, $P_{m}$ refuses the shares and outputs ver $_{m}=0$.

The reconstruct phase is the same as the previous scheme. Note that in this scheme the shared secret is

$$
s=\sum_{i \in \mathcal{L}} s_{i}
$$

In this scheme, each player in turn plays the part of the dealer. Thus the security of scheme follows from Theorem ' We need only to show that each good player has the same list $\mathcal{L}$, which is obvious.

Remark. As we indicated before, our VSS is modified from Blom's key predistribution scheme. In the original scheme, there is a dealer to construct the schemes. Using the methods of this section, we obtain a key predistribution scheme without dealer.

\section{New Proactive Scheme}

In this section, we describe our proactive secret sharing scheme without combinatorial structure. We will add combinatorial structures in this scheme to improve the efficiency of the scheme in next section.

\subsection{Initialization}

In the initial step, we assume that there is a dealer to set up the scheme. After the initialization phase, the dealer will no longer be needed.

In the initialization, we use the share phase of the VSS described in the Section $\mathbf{3}$ but we assume that $t>b+1$. The first four steps are the same. Then we do the following. 
5. If at least $n-b$ of the servers output $v r_{i}=1$, then the dealer erases all the information about the scheme on his end. Otherwise, the dealer reboots the whole system and initializes the system again.

\subsection{Share Renewal}

In the share renewal phase, all good servers do the following:

1. Each server $P_{l}$ selects a random symmetric polynomial

$$
r^{(l)}(x, y)=\sum_{i=0}^{t-1} \sum_{j=0}^{t-1} r_{i j} x^{i} y^{j}
$$

where $r_{00}=0$ and $r_{i j}=r_{j i}$ for all $i, j$.

2. $P_{l}$ sends $h_{k}^{(l)}(x)=r^{(l)}\left(x, \omega^{k}\right)$ to $P_{k}$ for $k=1,2, \cdots, n$ by a private channel and broadcasts $h_{0}^{(l)}(x)=r^{(l)}(x, 0)$.

3. $P_{k}$ checks whether $h_{0}^{(l)}(0)=0$ and $h_{k}^{(l)}(0)=h_{0}^{(l)}\left(\omega^{k}\right)$. If the conditions are satisfied, then $P_{k}$ computes and sends $P_{m}$ the value $h_{k}^{(l)}\left(\omega^{m}\right)$. Otherwise $P_{k}$ broadcasts an accusation of $P_{l}$.

4. $P_{m}$ checks whether $h_{m}^{(l)}\left(\omega^{k}\right)=h_{k}^{(l)}\left(\omega^{m}\right)$ for the values of $l$ not accused by $n-b$ servers of the system. If the equation is not true for more than $b$ values of $k$, then $P_{m}$ broadcasts an accusation of $P_{l}$.

5. If $P_{l}$ is accused by at most $b$ servers, then he can defend himself as follows. For those $P_{i}$ that $P_{l}$ is accused by, $P_{l}$ broadcasts $h_{i}^{(l)}(x)$. Then server $P_{k}$ checks whether $h_{i}^{(l)}\left(\omega^{k}\right)=h_{k}^{(l)}\left(\omega^{i}\right)$ and broadcasts "yes" or "no". If there are at least $n-b-2$ servers broadcasting yes, then $P_{l}$ is not a bad server.

6. $P_{m}$ updates the list of bad servers $\mathcal{L}$ by including all values $l$ for which $P_{l}$ is accused by at least $b+1$ servers, or found bad in the previous step. Then $P_{m}$ updates its shares as

$$
h_{m}(x) \longleftarrow h_{m}(x)+h_{m}^{(k)}(x)
$$

for all $k \notin \mathcal{L}$.

Remark. We can remove the private channels in step 2, since our scheme is also a key predistribution scheme and the server $P_{i}$ and $P_{j}$ can use $h_{i}(j)=h_{j}(i)$ as a key to communicate securely.

To check the security of the renewal phase, first we note that any coalition of at most $b$ servers cannot get any information about any shares except their own. In fact, a server $P_{i}$ only knows $h_{i}^{(l)}(x)$ and $h_{0}^{(l)}(x)$. Since $b<t-1$, the coalition of $b$ servers knows at most $t-1$ polynomials which cannot reveal $r^{(l)}(x, y)$ (see, e.g., 5). Secondly, from the protocol we know that every good server should have the same list $\mathcal{L}$. Therefore, the good servers will keep consistent shares after renewal. 
Note that a good server $P_{l}$ can be accused by at most $b$ servers. In this case, $P_{l}$ will broadcast $b$ polynomials in its defense. Thus $P_{l}$ will broadcasts total $b+1$ polynomials. Since $t>b+1$, these information will not reveal $r^{(l)}(x, y)$. On the other hand, suppose $P_{l}$ gives $P_{i}$ a wrong share, i.e., the share $P_{i}$ received is not consistent with at least $\frac{n-b}{2}$ other servers (the majority of good servers). Then $P_{i}$ will accuse $P_{l}$ in step 4 , since $\frac{n-b}{2}>b$. If $P_{l}$ broadcasts a correct share in the defense, then $P_{i}$ can correct his share. Otherwise, $P_{l}$ will be found to be bad.

\subsection{Recover a Share}

When a server is corrupted or replaced, it needs to be rebooted and thus it needs to recover the secret shares.

We first provide a protocol, to detect the corrupted servers, which we call detection.

Detection

1. $P_{l}$ computes and sends $h_{l}\left(\omega^{k}\right)$ to $P_{k}$ for $k=1,2, \cdots, n$ by private channels.

2. $P_{k}$ checks whether $h_{l}\left(\omega^{k}\right)=h_{k}\left(\omega^{l}\right)$. $P_{k}$ then broadcasts an accusation list $_{k}$ which contains those $l$ such that $h_{l}\left(\omega^{k}\right) \neq h_{k}\left(\omega^{l}\right)$ or $h_{l}\left(\omega^{k}\right)$ was not received.

3. Each good server updates the list $\mathcal{L}$ so that it contains those $l$ accused by at least $b+1$ servers of the system.

After running Detection, the system will recover the shares for all server $P_{l}$, where $l \in \mathcal{L}$. The recovery protocol is as follows.

1. For each $l \in \mathcal{L}$, every good server $P_{i}$ computes and sends $h_{i}\left(\omega^{l}\right)$ to $P_{l}$.

2. Upon receiving the data, $P_{l}$ computes a polynomial $h_{l}(x)$ such that $h_{l}\left(\omega^{k}\right)=$ $h_{k}\left(\omega^{l}\right)$ for the majority of $k$ it received, using the algorithms of $24 . P_{l}$ sets $h_{l}(x)$ as its shares.

\subsection{Reconstruct the Secret}

The reconstruction protocol is similar to the Reconstruction of VSS introduced in Section $\mathbf{3}$ We need only to change the first two steps as follows.

1' For each good server $P_{i}, P_{i}$ sends $h_{i}(0)$ to $P_{k}$, where $k$ is not in the list $\mathcal{L}$.

2' After receiving $h_{i}(0), P_{k}$ computes a polynomial $f_{k}(0, y)$ such that $f_{k}\left(0, \omega^{i}\right)=$ $h_{i}(0)$ for at least $n-2 b$ of the data he received.

\section{Combinatorial Structure}

In this section, we will introduce some combinatorial structure into our scheme. The combinatorial structure provides a predetermined arrangement of the servers which permits the possibility of reducing the computation of the scheme. 


\subsection{Set Systems}

A set system is a pair $(X, \mathcal{B})$, where $X$ is a set of $n$ points and $\mathcal{B}$ is a collection of subsets of $X$ called blocks.

We will use a set system with the following properties, where $t \leq \frac{n}{4}-1$ :

1. $|B| \geq t$ for any $B \in \mathcal{B}$.

2. For any subset $F \subset X$ with $|F| \leq b$, there exists a $B \in \mathcal{B}$ such that $F \cap B=\emptyset$.

It is easy to see that such a set system exists. For example, we can choose $\mathcal{B}$ to be all the $t$-subsets of $X$. However, there are often better set systems (i.e., set systems containing fewer blocks). The following definition is well-known (see, e.g., 24 ).

Definition $3 A$ collection $\mathcal{T}$ of $k$-subsets of $\{1, \ldots, n\}$ (called blocks) is an $(n, k, b)$-covering if every $b$-subset of $\{1, \ldots, n\}$ is contained in at least one block.

It is easy to see that if $(X, \mathcal{T})$ is an $(n, n-t, b)$-covering, then the set system

$$
\{\{1, \ldots, n\} \backslash T: T \in \mathcal{T}\}
$$

is a set system satisfying our purpose. There are several efficient constructions of $(n, n-t, b)$-coverings in 24 which can be easily implemented by a computer.

\subsection{Applying Set System to the Proactive VSS}

The idea of using the set system is to reduce the computations for the share renewal and share recover protocols. In the scheme of Section 4 share renewal and share recover used the data from all the participants. However, these operations can be carried out using the data from $t$ good servers. For example, in share renewal protocol, any $t$ good servers can renew the shares, since the shares are polynomials of degree at most $t-1$. In protocol of Section 4 every good server provides information to renew shares. So there are redundant computations. If the system can determine $t$ good servers, then the protocol will be more efficient. Note that there are at least $3 t+1$ good servers in the system. Thus we can save at least one third of the computations. On the other hand, we should be very careful when the $t$ good servers are selected, since the adversary is mobile. The good server could turn to bad at any time. Thus in the scheme of this section, we will actually select correct information instead of good servers, although we will still use "good server" for convenience.

Now let us use the set system to improve our proactive scheme. Suppose $(X, \mathcal{B})$ is a set system satisfying the conditions of subsection 5 ll where $X=$ $\{1,2, \cdots, n\}$, and $\mathcal{B}=\left\{B_{1}, B_{2}, \cdots, B_{s}\right\}$. The set system is published so that each participant can consult it.

Note that in our scheme, in any phase there is a list $\mathcal{L}$ containing all the bad servers. By the property of the set system, there is a block $B$ which contains only good servers. If the system can determine one of the "good" blocks, then 
the system can renew the shares or recover the shares only using the data from these servers. We will call these servers the members of an executive committee.

For a list $\mathcal{L}$ of bad servers, the system can decide following list of blocks: $B_{i_{1}}, B_{i_{2}}, \cdots, B_{i_{e}}$, such that $B_{i_{j}} \cap \mathcal{L}=\emptyset, j=1,2, \cdots, e$, and $1 \leq i_{1}<i_{2}<\cdots<$ $i_{e} \leq s$. These blocks are called executive committee candidates. Note that the adversary is mobile, therefore we cannot guarantee that these candidates contain only good servers in the next time period.

The proactive secret sharing scheme with combinatorial structure works as follows. The initialization is the same as that in Section 1 In each time period the system does the following.

1. Run Detection to obtain the list $\mathcal{L}$ of bad servers and the executive committee candidates: $B_{i_{1}}, B_{i_{2}}, \cdots, B_{i_{e}}$.

2. If an executive committee has not been found, then for next executive committee candidate $B$, each $P_{g} \in B$ does the following:

(a) Selects a random symmetric polynomial

$$
r^{(g)}(x, y)=\sum_{i=0}^{t-1} \sum_{j=0}^{t-1} r_{i j} x^{i} y^{j},
$$

where $r_{00}=0$ and $r_{i j}=r_{j i}$ for all $i, j$, and sends $h_{k}^{(g)}(x)=r^{(g)}\left(x, \omega^{k}\right)$ to $P_{k}$ for $k=1,2, \cdots, n, k \neq g$ by private channel and broadcasts $h_{0}^{(g)}(x)=$ $r^{(g)}(x, 0)$.

(b) $P_{k}$ checks whether $h_{0}^{(g)}(0)=0$ and $h_{k}^{(g)}(0)=h_{0}^{(g)}\left(\omega^{k}\right)$ for $g \in B$. If the conditions are satisfied, then $P_{k}$ computes and sends $P_{m}$ the value $h_{k}^{(g)}\left(\omega^{m}\right)$. Otherwise $P_{k}$ broadcasts an accusation of $P_{g}$.

(c) $P_{m}$ checks whether $h_{m}^{(g)}\left(\omega^{k}\right)=h_{k}^{(g)}\left(\omega^{m}\right)$ for $g \in B$. If the equation is not true, then $P_{m}$ broadcasts an accusation of $P_{g}$.

(d) A member in $B$ is accused by at least $b+1$ servers is bad. If a member in $B$ is accused by at most $b$ servers, then it can defend itself. If no member in $B$ is bad, then $B$ is found to be the executive committee.

3. The system runs the recovery protocol to recover the shares for the servers in $\mathcal{L}$.

4. Each server $P_{m}$ updates its shares as

$$
h_{m}(x) \longleftarrow h_{m}(x)+h_{m}^{(g)}(x)
$$

for all $g \in B$.

The reconstruction protocol is the same as that in Section 7

\subsection{Applying Combinatorial Structures to Other Schemes}

The proactive secret sharing scheme proposed by Herzberg et al. in I. 5 is similar to our scheme in Section 1 Thus it is straightforward to modify our method with combinatorial structures to their scheme. In general, suppose a proactive secret sharing scheme has the following properties: 
1. Information from any $t$ good servers can be used to renew shares and recover shares.

2. A VSS exists in which any server can use this VSS to send data which can be verified by the system.

3. There is a detection protocol to find the bad servers.

4. There is a defense protocol so that an accused server can be determined bad or good by the system.

5. There are renewal and share recovery protocols.

6. There is set system $(X, \mathcal{B})$ satisfying the conditions of Subsection 5.

Then we can use the following scheme for renewal and share recovery protocols.

1. Run the detection protocol to obtain a list $\mathcal{L}$ of bad servers and the executive committee candidates: $B_{i_{1}}, B_{i_{2}}, \cdots, B_{i_{e}}$.

2. If executive committee has not been found, then for next executive committee candidate $B$, each $P_{g} \in B$ does:

(a) Send recovery information $r c_{k}^{g}$ to $P_{k}$ for each $k \in \mathcal{L}$ in the system and send renewal information $r n_{l}^{g}$ to $P_{l}$ for each $l$ in the system by VSS.

(b) The system checks the correctness of $r c_{k}^{g}$ and $r n_{l}^{g}$. If some mistake is found, then $P_{g}$ is accused.

(c) A member in $B$ is accused, then it can defend itself and the system can decide whether it is bad. If no member in $B$ is bad, then $B$ is defined to be the executive committee.

3. The $P_{k} \in \mathcal{L}$ recovers its share using $\left\{r c_{k}^{g}: g \in B\right\}$.

4. Each server $P_{l}$ renews its share using $\left\{r n_{l}^{g}: g \in B\right\}$.

It is readily checked that the proactive secret sharing scheme of 15 satisfies all the properties we needed. Thus we can use the combinatorial method to improve their scheme. The details are omitted here.

\section{Acknowledgment}

The authors thank K. Kurosawa for his valuable comments.

\section{References}

1. N. Alon, Z. Galil and M. Yung, Efficient dynamic-resharing "verifiable secret sharing" against mobile adversary, European Symposium on Algorithms (ESA) 95, LNCS 979, 523-537.

2. J. C. Benaloh, Secret sharing homomorphisms: keeping shares of a secret secret, Advances in Cryptology-Crypto'86, LNCS 263, 1987, 251-260.

3. M. Ben-Or, S. Goldwasser and A. Wigderson, Completeness Theorems for Noncryptographic Fault-Tolerant Distributed Computations, Proc. 20th Annual Symp. on the Theory of Computing, ACM, 1988, 1-10.

4. G.R. Blackley, Safeguarding cryptographic keys. Proc. Nat. Computer Conf. AFIPS Conf. Proc., 1979, 313-317. 
5. R. Blom, An optimal class of symmetric key generation systems, Eurocrypt'84, LNCS 209, (1985), 335-338.

6. R. Canetti and A. Herzberg, Maintaining security in the presence of transient faults, Crypto'94, LNCS 839, 1994.

7. D. Chaum, C. Crepeau and I. Damgard, Multiparty Unconditionally Secure Protocols, Proc. 20th Annual Symp. on the Theory of Computing, ACM, 1988, 11-19.

8. B. Chor, S. Goldwasser, S. Micali and B. Awerbuch, Verifiable Secret Sharing and Achieving Simultaneity in Presence of Faults, Proc. 26th Annual Symp. on the Foundations of Computing Science, IEEE, 1985, 383-395.

9. P. Feldman, A Practical Scheme for Non-Interactive Verifiable Secret sharing, Proc. 28th Annual Symp. on the Foundations of Computing Science, IEEE, 1987, 427437.

10. P. Feldman and S. Micali, An Optimal Algorithm for Synchronous Byzantine Agreement, Proc. 20th Annual Symp. on Theory of Computing, ACM, 1988, 148161.

11. Y. Frankel, P. Gemmel, P. D. MacKenzie and M. Yung, Proactive RSA, Crypto'97, LNCS 1294, 440-452.

12. R. Gennaro, M. O. Rabin and T. Rabin, Simplified VSS and fast-track multiparty computations with applications to threshold cryptography, Proc. of 17th ACM Symp. on Principles of Distributed Computing, (1998), 101-111.

13. O. Goldreich, S. Micali and A. Wigderson, Proofs that Yield Nothing But Their Validity or All Languages in NP Have Zero-Knowledge Proof Systems, Journal of the ACM, 38(1991), 691-729.

14. A. Herzberg, M. Jakobsson, S. Jarecki, H. Krawczyk and M. Yung, Proactive public key and signature systems, The 4th ACM Symp. on Comp. and Comm. Security, April 1997.

15. A. Herzberg, S. Jarecki, H. Krawczyk and M. Yung, Proactive secret sharing or: How to cope with perpetual leakage, Crypto'95, LNCS 963339-352.

16. I. Ingemarsson and G. J. Simmons, A protocol to set up shared secret schemes without the assistance of a mutually trusted party, Eurocrypt'90, LNCS 473, 1990, 266-282.

17. R. J. McEliece and D. V. Sarwate, On Sharing Secrets and Reed-Solomon Codes, Communications of the ACM, 24(1981), 583-584.

18. R. Ostrovsky and M. Yung, How to withstand mobile virus attacks, ACM Symposium on principles of distributed computing, 1991, 51-59.

19. T. P. Pedersen, Non-interactive and information-theoretic secret sharing, Advances in Cryptology - Crypto'91, LNCS 576, 1991, 129-140.

20. T. Rabin, Robust sharing of secrets when the dealer is honest or faulty, Journal of the ACM, 41(1994), 1089-1109.

21. T. Rabin, A simplified approach to threshold and proactive RSA, Crypto'98, LNCS 1462, 1998, 89-104.

22. T. Rabin and M. Ben-Or, Verifiable secret sharing and multiparty protocols with honest majority, Proc. 21st Annual Sympo. on the Theory of Computing, ACM, 1989, 73-85.

23. A. Shamir, How to share a secret, Commun. ACM, 22(1979), 612-613.

24. R. S. Rees, D. R. Stinson, R. Wei and G. H. J. van Rees, An application of covering designs: determining the maximum consistent set of shares in a threshold scheme, Ars Combin., to appear.

25. D. R. Stinson, Cryptography Theory and Practice, CRC Press, 1995.

26. M. Tompa and H. Woll, How to share a secret with cheaters, Journal of Cryptology, 1(1988), 133-138. 